\title{
Novel wavelength tunable filter offering multi-stage selection for colorless, directionless, and contentionless ROADMs
}

\author{
Tomonobu Niwa ${ }^{1 a)}$, Hiroshi Hasegawa ${ }^{1}$, Ken-ichi Sato ${ }^{1}$, \\ Toshio Watanabe ${ }^{2 b)}$, Hiroshi Takahashi ${ }^{2 \mathrm{c})}$, \\ and Masayuki Okuno ${ }^{3 \mathrm{~d}}$ ) \\ ${ }^{1}$ Department of Electrical Engineering and Computer Science, Nagoya University \\ Furo-cho, Chikusa-ku, Nagoya, 464-8603 Japan \\ ${ }^{2}$ NTT Photonics Laboratories \\ ${ }^{3}$ NTT Electonics Corporation \\ a)tomono_n@echo.nuee.nagoya-u.ac.jp \\ b)w.toshio@lab.ntt.co.jp \\ c) t.hiroshi@lab.ntt.co.jp \\ d) okuno-m@ntt-el.com
}

\begin{abstract}
We demonstrate a Colorless, Directionless, and Contentionless add/drop that is made possible by newly proposed multistage tunable filters; it will be effective in the creation of flexible and dynamic optical path networks. The filters consist of small-scale switches and AWGs (Arrayed Waveguide Gratings) and so are cost effective. The technical feasibility of the drop configuration using proposed tunable filters is experimentally verified.
\end{abstract}

Keywords: optical path network, arrayed waveguide grating, tunable filter, colorless, directionless, contentionless

Classification: Optoelectronics, Lasers and quantum electronics, Ultrafast optics, Silicon photonics, Planar lightwave circuits

\section{References}

[1] K. Sato, "OOO switching - the role and technological advances," Proc. Opt. Fiber Commun. Nat. Fiber Opt. Engr. Conf. (OFC/NFOEC), Paper NThB1, March 2011.

[2] V. Kaman, R. J. Helkey, and J. E. Bowers, "Multi-degree ROADM's with agile add-drop access," Proc. Photonics in Switching, Paper TuA2.5, Aug. 2007.

[3] R. A. Jensen, "Optical switch architecture for emerging colorless/ directionless/contentionless ROADM networks," Proc. Opt. Fiber Commun. Nat. Fiber Opt. Engr. Conf. (OFC/NFOEC), Paper OThR3, March 2011.

[4] P. N. Ji and Y. Aono, "Colorless and directionless multi-degree reconfigurable optical add/drop multiplexers," Proc. Wireless and Opt. Commun. 
Conf. (WOCC), pp. 13-17, May 2010.

[5] T. Watanabe, K. Suzuki, T. Goh, K. Hattori, A. Mori, T. Takahashi, T. Sakamoto, K. Morita, S. Sohma, and S. Kamei, "Compact PLC-based transponder aggregator for colorless and directionless ROADM," Proc. Opt. Fiber Commun. Nat. Fiber Opt. Engr. Conf. (OFC/NFOEC), Paper OTuD3, March 2011.

[6] B. C. Collings, "Wavelength selectable switches and future photonic network applications," Proc. Photonics in Switching, Paper FrII2-4, Sept. 2009.

[7] L. E. Nelson, S. L. Woodward, P. D. Magill, S. Foo, M. Moyer, and M. O'Sullivan, "Real-time detection of a $40 \mathrm{Gbps}$ intradyne channel in the presence of multiple received WDM channels," Proc. Opt. Fiber Commun. Nat. Fiber Opt. Engr. Conf. (OFC/NFOEC), Paper OMJ1, March 2010.

[8] N. Kikuchi, Y. Shibata, H. Okamoto, Y. Kawaguchi, S. Oku, Y. Kondo, and Y. Tohmori, "Monolithically integrated 100-channel WDM channel selector employing low-crosstalk AWG," IEEE Photon. Technol. Lett., vol. 16, no. 11, pp. 2481-2483, Nov. 2004.

[9] R. Hirako, K. Ishii, H. Hasegawa, K. Sato, and O. Moriwaki, "Compact matrix-switch-based hierarchical optical path cross-connect with colorless waveband add/drop ratio restriction," IEICE Trans. Commun., vol. E94B, no. 4, pp. 918-927, April 2011.

\section{Introduction}

To cope with exploding Internet traffic demand, optical path networks using Reconfigurable Optical Add/Drop Multiplexers (ROADMs) are being widely introduced around the world. The networks provide the advantages of large capacity transmission and low electric power consumption as compared to networks using electric routing systems [1]. Although current ROADMs are mostly operated in a static manner, dynamic path operations will be required to provide large bandwidth on demand services and/or reduce network operation cost by minimizing necessary human intervention in accommodating traffic demands. Dynamic optical path allocation requires flexible bridging capability between any arbitrary selected add/drop wavelength path and any transponder, which is possible with the so-called Colorless, Directionless, and Contentionless $(\mathrm{C} / \mathrm{D} / \mathrm{C})$ capabilities [2]. Colorless function signifies that any wavelength path in a fiber can access a transponder. Directionless function allows any transponder to be connected to any input/output port of the optical node system. Contentionless function denotes that any combination of wavelength path and transponder can be connected in a non-blocking manner at the add/drop portion. Cost effectiveness, compactness, fine modular growth capability, scalability, and reliability are desirable attributes that $\mathrm{C} / \mathrm{D} / \mathrm{C}$ add/drop systems should satisfy. We propose here a novel device that effectively embodies the $\mathrm{C} / \mathrm{D} / \mathrm{C}$ add/drop architecture; its technical feasibility is experimentally verified. 


\section{$2 \mathrm{C} / \mathrm{D} / \mathrm{C}$ add/drop systems with wavelength tunable filters}

Let $K$ be the number of input/output fibers of an ROADM, $L$ be the number of wavelength paths per fiber, and $\mathrm{M}(\leq K \times L)$ be the maximum number of added or dropped wavelength paths that denote originating or terminating wavelength paths. Different $\mathrm{C} / \mathrm{D} / \mathrm{C}$ add/drop architectures have been discussed so far. They can be classified into two types; matrix switch based [3, 4] and broadcast \& select based [5]. Hybrid variants are also possible. The matrix switch type architecture utilizes relatively large-scale matrix switches to realize flexible routing in add/drop parts. For example, a $320 \times 160$ matrix switch will be necessary when $K=8, L=40, M=160$, but is hard to be realized cost-effectively. The broadcast \& select type is composed of optical splitters and wavelength path selection devices. The cost of optical splitters is relatively small and the splitting loss can be compensated by amplifiers. Hence, the key is the wavelength path selection device, especially at the drop side. From the viewpoints of simplicity, cost, and modular growth capability, one promising approach will be to combine small-size switches and tunable filters as shown in Fig. 1. Please note that multiple stage Wavelength Selective Switch (WSS) architecture does not attain contentionless capability [6]. At the add part (see Fig. 1 lower right insertion), the interconnection between $1 \times M$ splitters and $1 \times K$ switches allows an optical signal from each transmitter to be injected into any of $K$ output fibers. Similarly at the drop part side, the combination of $1 \times M$ splitters and $1 \times K$ switches followed by a tunable filter allows any wavelength from any $K$ input fibers to be dropped and connected to an optical receiver. To realize the tunable filter, one approach is to utilize $1 \times 1 \mathrm{WSSs}$ and another is to apply coherent detection [7], however, both tend to be complex and costly to implement. The power loss of the splitters is compensated by inserting an Erbium-Doped optical Fiber Amplifier (EDFA) at an appropriate location. When one EDFA is inserted
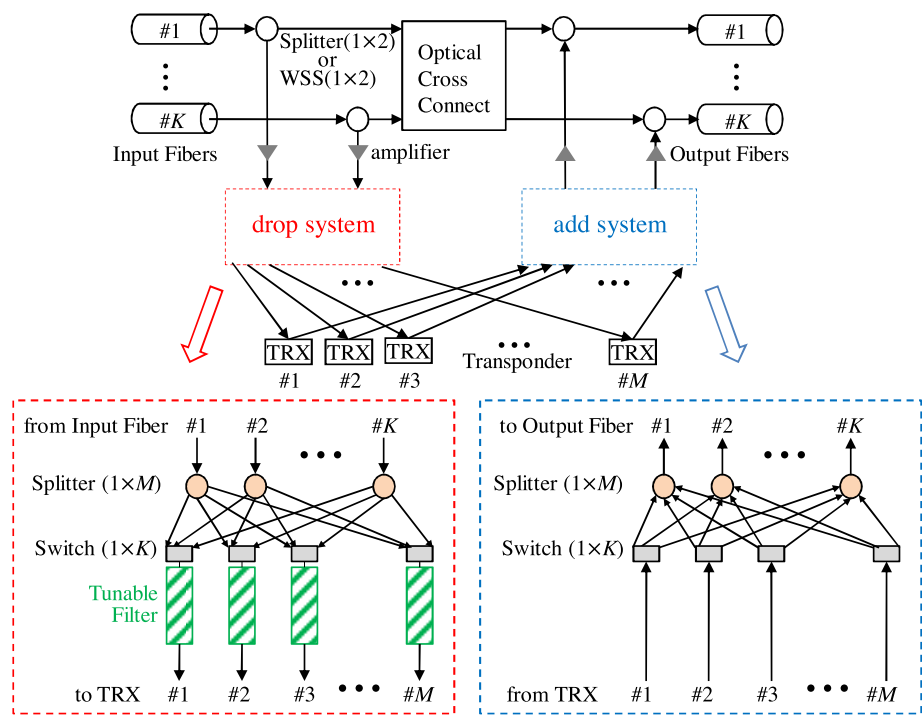

Fig. 1. Add/drop architecture utilizing proposed tunable filters 
for each incoming line fiber to be dropped, $K$ EDFAs are required. The $K$ EDFAs are shared by $M$ drop ports, and hence the EDFA cost per one drop port can be rather small. The $1 \times M$ splitters and the $1 \times K$ switches can be easily manufactured with existing technologies. The portion can be developed with individual devices or as integrated devices as reported by [5]. As a result, the tunable filter is the key to make this architecture effective. The add/drop architecture provides good modular growth capability or pay-asyou-grow capability. Our proposed novel tunable filter is shown in the next section.

\section{Proposed tunable filter based on multi-stage wavelength se- lection}

A straightforward approach to realize a tunable filter is to connect a $1 \times$ $L$ Arrayed Waveguide Grating (AWG) with an $L \times 1$ switch, however, the switch scale is large; $L$ is 96 , for example. We, therefore, developed a novel wavelength tunable filter based on multi-stage wavelength selection. In each stage, we use a demultiplexer, cyclic AWG, for dividing a set of incoming wavelengths into groups, and a switch for selecting any one of the groups. The number of wavelengths is reduced as they pass through each stage. In the last stage, just the desired wavelength is output.

Hereafter, we evaluate necessary switch scale in terms of the number of $1 \times$ 2 switch points. $J \times(\sqrt[J]{L}-1)$ is the minimum switch scale of a $J$-stage tunable filter where each stage consists of a $1 \times$ (multi-output) AWG and a switch. This formulation ignores a detailed requirement on the output port number of each AWG (explained later), however, it yields a good approximation. The degree of the switch scale reduction attained by incrementing the number of stages beyond 3 is small. For example, in the case of $L=40$, while the 1 -stage filter configuration needs a $40 \times 1$ switch that consists of $40-1=391 \times 2$ switch elements. The 2 -stage architecture, in which the output port numbers of the first cyclic AWG and the second cyclic AWG are 5 and 8, respectively, needs $(5-1)+(8-1)=11$ switch elements. Similarly, the 3 -stage architecture requires $(3-1)+(4-1)+(5-1)=9$ switch elements. The 2 -stage filter achieves a $72 \%$ switch scale reduction compared to the equivalent 1-stage filter. However, the 3 -stage filter achieves $77 \%$ reduction compared to the 1-stage filter, and the improvement over the 2-stage filter is marginal. In addition, the more stages there are, the greater the optical loss becomes because the optical signals pass through more switches and AWGs. We, therefore, the adopted 2-stage filter architecture.

A similar but different architecture was previously proposed [8] and a 100channel WDM channel selector was fabricated. It consists of two stages of AWGs, two stages of Semiconductor Optical Amplifier (SOA) gates and a 10× 1 coupler. The configuration uses amplifiers at each stage, which degrades the signal-to-noise ratio. Our proposed architecture uses high-resolution AWGs and does not need amplifiers for each stage. This simplifies the architecture and contributes to the attainment of high performance. 


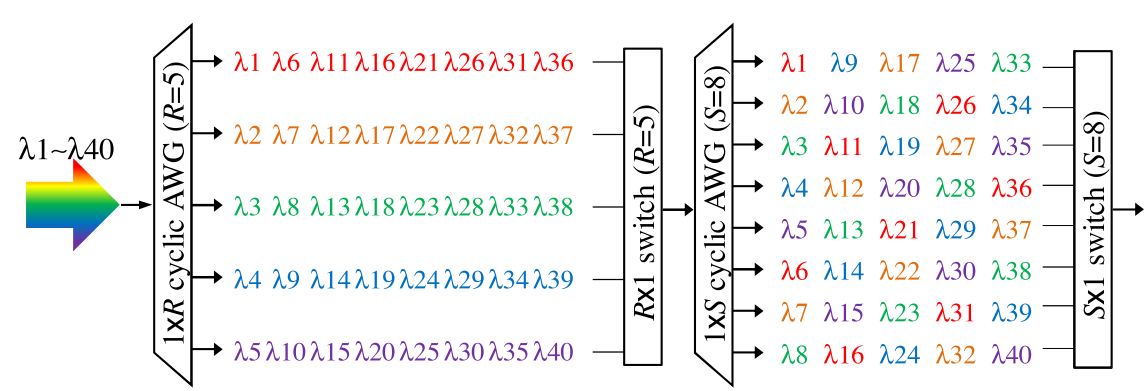

(a) A basic 2-stage tunable filter

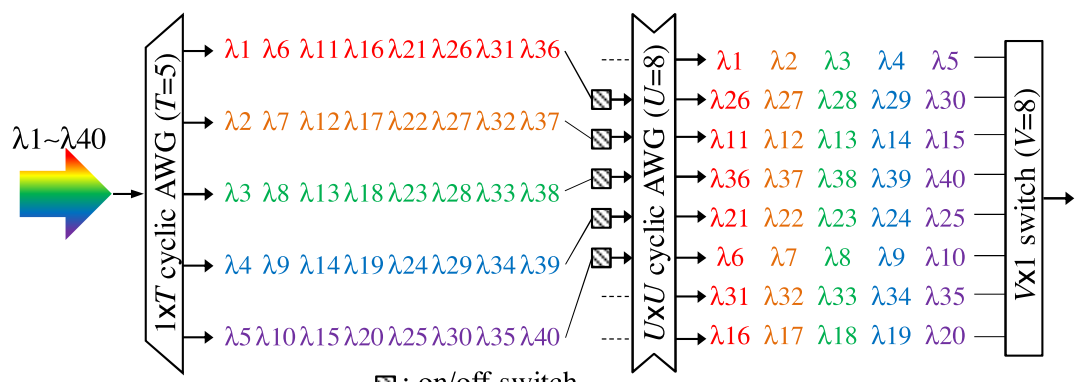

(b) An alternative 2-stage tunable filter

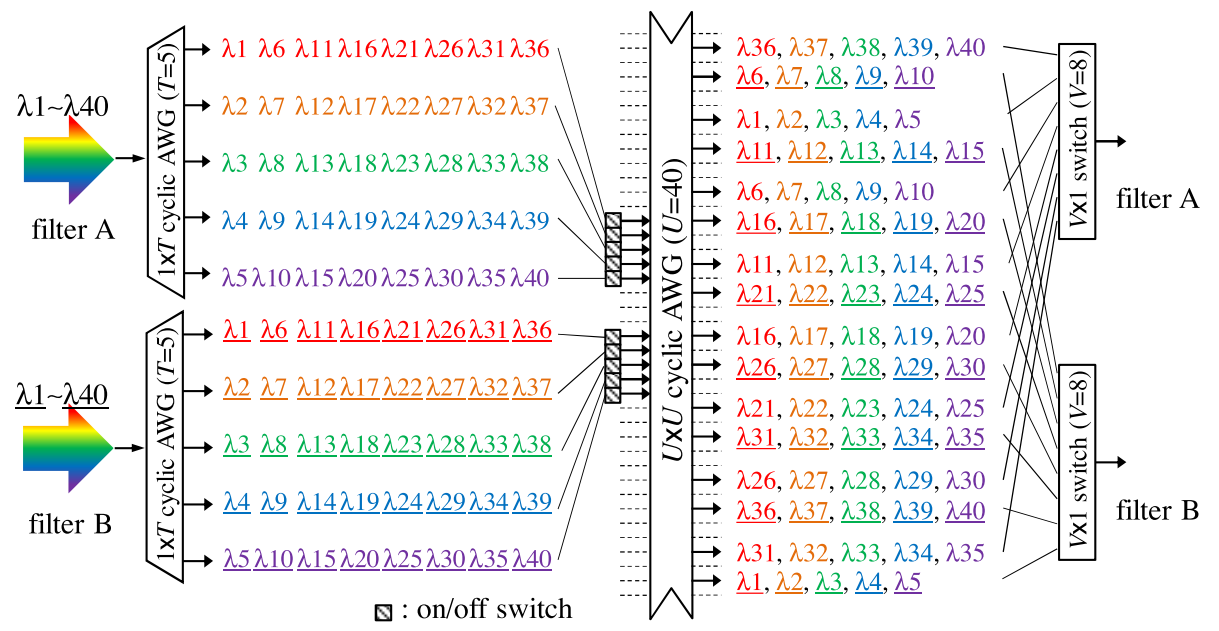

(c) A configuration aggregated multiple 2-stage filters

Fig. 2. Novel 2-stage tunable filter configurations

Proposed multi-stage tunable filters can be fabricated with various combinations of demultiplexers and switches. Fig. 2 depicts three examples of our proposed 2-stage tunable filter configuration. Parameter $\delta f$ represents the frequency interval on WDM channels. Fig. 2 (a) shows a filter comprised of a $1 \times R$ cyclic AWG with channel spacing $\delta f$ and FSR (Free Spectral Range) $R \delta f$ and an $R \times 1$ switch for the first stage, and a $1 \times S$ cyclic AWG with channel spacing $\delta f$ and FSR $S \delta f$ ) and a $S \times 1$ switch for the second stage. This configuration must meet the requirement that the least common multiple of $R$ and $S$ is equal to or larger than $L$. This is because the wavelengths are output at intervals of $R$ from an output port of the $1 \times R$ AWG, and the wavelengths are output at intervals of $S$ from an output port of the $1 \times S \mathrm{AWG}$, and therefore, to prevent the overlapping of output wavelengths 
at any output of the second AWG, the least common multiple of $R$ and $S$ must be equal to or larger than $L$.

The other examples shown in Figs. $2(\mathrm{~b})$ and (c) combine a $1 \times T$ cyclic AWG with channel spacing $\delta f$ and FSR $T \delta f$ and $T$ on/off switches for the first stage, and a $U \times U$ cyclic AWG with channel spacing $\delta f$ and FSR $U \delta f$ and a $V \times 1$ switch $(V \leq U)$ for the second stage.

Using multiple tunable filters to allow component sharing will enhance the cost effectiveness. Fig. 2 (c) depicts an advanced filter configuration where a $40 \times 40$ cyclic AWG for the second stage is connected to two $1 \times 5$ cyclic AWGs; in this example, two filters (filter A and B) use a common $40 \times 40$ AWG as their second stage. Up to five filters can share one $40 \times 40$ AWG in the same matter. This configuration will enable us to efficiently integrate multiple filters in a compact way, for example, on a single PLC (Planar Lightwave Circuit) chip.

\section{Prototype and transmission experiments}

In order to confirm the technical feasibility of the proposed system, we tested the 2-stage filter architecture shown in Fig. 2 (c). The prototype developed in this work consists of several components; 4 arrayed $1 \times 5$ cyclic AWGs (channel spacing $=100 \mathrm{GHz}$ and $\mathrm{FSR}=500 \mathrm{GHz}$ ), a $40 \times 40$ cyclic AWG [9], and switches. The integration of multiple $1 \times 5$ AWGs is important for compact and cost effective implementation. These AWGs are utilized independently for the proposed tunable filters while the $40 \times 40$ AWG can be shared by multiple filters. Figs. 3 (a) and (b) plot the WDM signals consisting of

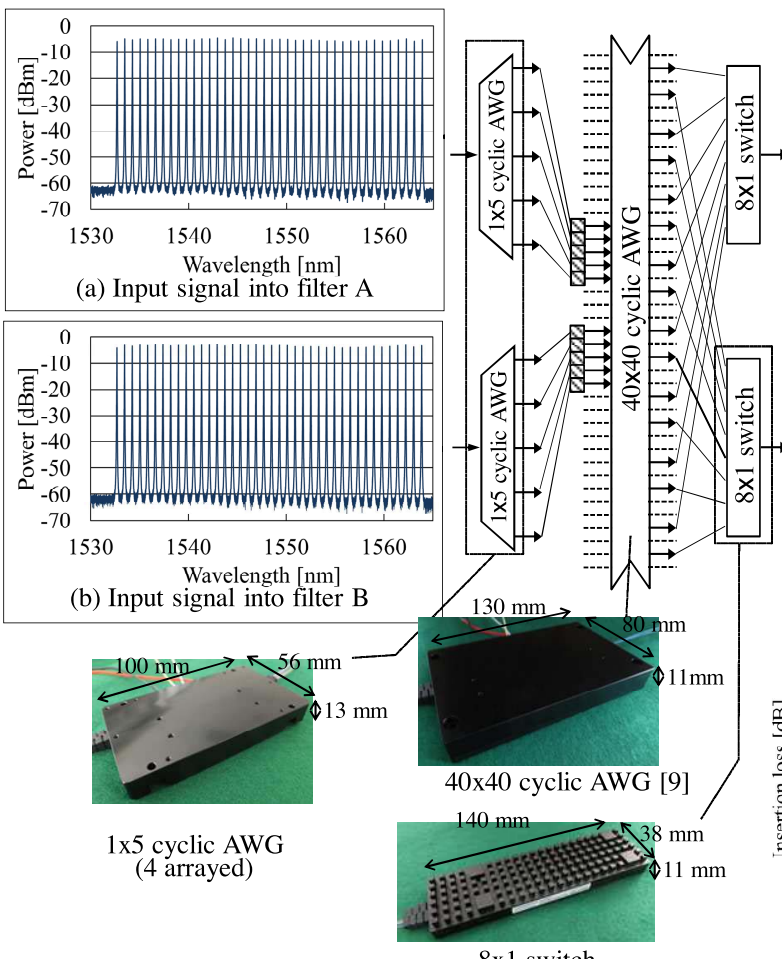

$8 \times 1$ switch

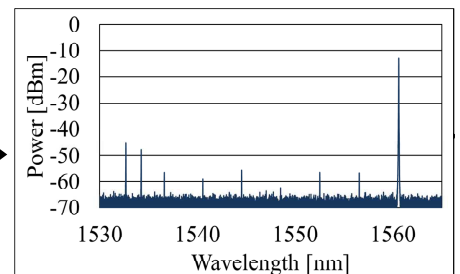

(c) Output spectrum from filter A ( $\lambda 36)$
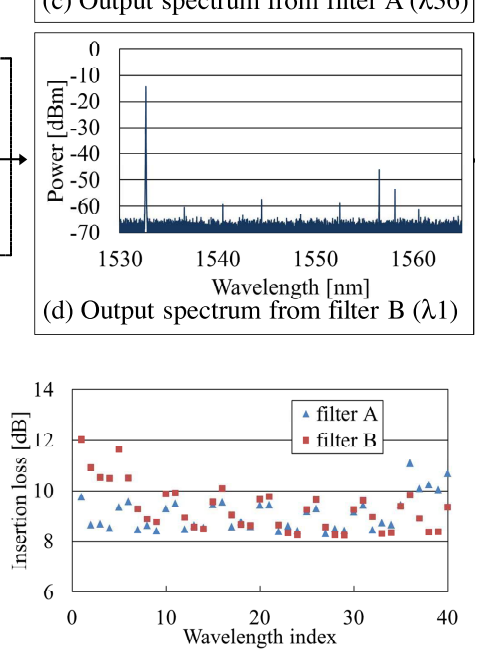

(e) Loss variation

Fig. 3. Experimental results of the prototype filter 
40 channels with $100 \mathrm{GHz}$ spacing $(191.7+0.1 \times n \mathrm{THz} ; n=0 \sim 39)$ on the ITU-T grid. The signals were simultaneously input to filters A and B (see Fig. 2 (c)). Figs. 3 (c) and (d) provide examples of extracted output channels when the target channels are set at $\lambda 36=1560.60 \mathrm{~nm}$ and $\lambda 1=1532.68 \mathrm{~nm}$, respectively. We can verify that each selected channel was successfully extracted in each experiment. The loss variations are shown in Fig. 3 (e). The variation stems from the $40 \times 40 \mathrm{AWG}$ since the deviation of passband to the ITU-T grid increases with the AWG size; moreover, some wavelengths are in a different FSR index, which increases the loss.

\section{Conclusions}

In order to minimize manual operation and accommodate future dynamic optical path services, ROADMs with dynamic add/drop operation capabilities are critical. To enable this, we proposed an efficient Colorless, Directionless, and Contentionless add/drop system that utilizes a novel tunable filter architecture. The proposed tunable filter is realized by exploiting a multi-stage selection configuration and as a result it is realized with a relatively small number of switch elements. Furthermore, it is shown that the second stage AWG can be shared by multiple filters, which is expected to enhance degree of integration. We developed a prototype device and verified its performance. The filter will provide a viable solution in cost-effectively realizing $\mathrm{C} / \mathrm{D} / \mathrm{C}$ add/drop capability with excellent modular growth characteristics.

\section{Acknowledgments}

This works was partly supported by CREST and KAKENHI (23246072). 